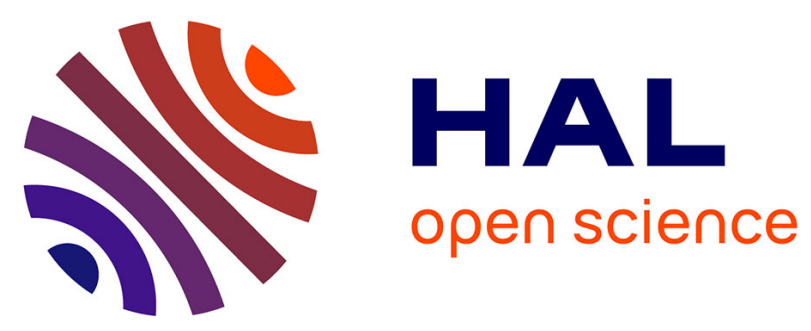

\title{
A methodology to obtain data at the slip-band scale from atomic force microscopy observations and crystal plasticity simulations. Application to hydrogen-induced slip localization on AISI 316L stainless steel
}

Isabelle Aubert, Nicolas Saintier, Jean-Marc Olive, François Plessier

\section{To cite this version:}

Isabelle Aubert, Nicolas Saintier, Jean-Marc Olive, François Plessier. A methodology to obtain data at the slip-band scale from atomic force microscopy observations and crystal plasticity simulations. Application to hydrogen-induced slip localization on AISI 316L stainless steel. Acta Materialia, 2016, 104, pp.9-17. 10.1016/j.actamat.2015.11.042 . hal-01344808

\author{
HAL Id: hal-01344808 \\ https://hal.science/hal-01344808
}

Submitted on 12 Jul 2016

HAL is a multi-disciplinary open access archive for the deposit and dissemination of scientific research documents, whether they are published or not. The documents may come from teaching and research institutions in France or abroad, or from public or private research centers.
L'archive ouverte pluridisciplinaire $\mathbf{H A L}$, est destinée au dépôt et à la diffusion de documents scientifiques de niveau recherche, publiés ou non, émanant des établissements d'enseignement et de recherche français ou étrangers, des laboratoires publics ou privés. 


\title{
A methodology to obtain data at the slip-band scale from atomic force microscopy observations and crystal plasticity simulations. Application to hydrogen-induced slip localization on AISI 316L stainless steel
}

\author{
Isabelle Aubert ${ }^{\mathrm{a},{ }^{*}}$, Nicolas Saintier ${ }^{\mathrm{b}}$, Jean-Marc Olive ${ }^{\mathrm{c}}$, Francois Plessier ${ }^{\mathrm{a}}$ \\ ${ }^{a}$ Université de Bordeaux, I2M, UMR 5295, 351 cours de la Libération, 33405 Talence Cedex, France \\ ${ }^{\mathrm{b}}$ Arts et Métiers ParisTech (centre de Bordeaux-Talence), I2M, UMR 5295, Esplanade des Arts et Métiers 33405 Talence Cedex, France \\ ${ }^{\mathrm{c}}$ CNRS, I2M, UMR 5295, 351 cours de la Libération, 33405 Talence Cedex, France
}

Keywords:

Finite element calculation

Crystal plasticity

Atomic force microscopy

Slip band

Hydrogen

\begin{abstract}
A B S T R A C T
A local approach coupling atomic force microscopy (AFM) observations and polycrystal finite element calculations is proposed to provide data at the slip-band scale. From AFM measurements of slip bands emerging at the specimen surface during tensile loading, and from numerical results of strain fields at the grain scale, the method is able to determine at the local discrete scale the mean slip-band spacing and the number of dislocations emerging during the plastic strain. The methodology applied in the hydrogen embrittlement context highlights, quantitatively, at the grain scale an increased plastic strain localization with internal hydrogen.
\end{abstract}

\section{Introduction}

In the case of austenitic stainless steels, hydrogen-plasticity interaction can lead to various kinds of degradation as a severe ductility reduction associated with a brittle-like intergranular and transgranular cracking [1]. At the microscopic scale, transmission electron microscopy observations highlighted the effect of hydrogen on the dislocation mobility, the dislocation density in pile-ups, and other mechanisms such as cross slip [2]. At the mesoscopic scale, correlations between slip-band emergence and dislocation pattern were clearly established $[3,4]$. In a previous statistical study, slip-band feature, observed using atomic force microscopy (AFM), have been used to provide a quantitative analysis of the effect of hydrogen on plastic strain localization [5] of strained AISI 316L stainless steel samples as a function of the macroscopic plastic strain level, the grain size and the hydrogen content. However a more important effect of hydrogen uptake

\footnotetext{
* Corresponding author.

E-mail address: i.aubert@i2m.u-bordeaux1.fr (I. Aubert).
}

could be masked by mechanical field heterogeneities that develop in polycrystals under tension due to the geometry and crystallographic orientation of grains. Such an heterogeneity induces a very different response to the hydrogen absorption from one grain to another [6]. In this work, a local approach coupling atomic force microscopy measurements and polycrystal finite element calculations is proposed to obtain data at the slip-band scale.

Crystal plasticity finite element model is used to calculate intra and intergranular heterogeneities of mechanical fields. There is a good agreement between the 3-D computation results, based on the mesh of the real 3-D microstructure, and the local strain field measurements [7]. The results, obtained at the continuous mesoscopic scale can highlight the deformation incompatibilities at the grain boundaries. But this scale of modeling prevents any direct comparison with measurements obtained from local techniques such as AFM [3,8-11] and EBSD [12-14] which provide informations at the discrete scale. Indeed, crystal plasticity finite element model correctly estimates the shear intensity and the slip system activation, but the strain heterogeneity location may differ from AFM-measurements [15], and it cannot specify the slip band position, nor their height and spacing. Thus, a numerical strain field 
can match with several morphologies observed by AFM (Fig. 1). A new methodology is proposed to overcome this limitation: The AFM results are used to describe the behavior of the average individual slip-bands and the numerical results provide the state of plasticity observed at the scale of continuous slip systems and are used to interpret the AFM results.

Before the description of experimental and numerical methods, the influence of the subsurface geometry of grains is investigated. Then, the coupling between AFM measurements of slip bands emerging at the stainless steel surface during the plastic strain, and numerical results of strain fields at the grain scale is presented. Finally, the methodology is applied in the hydrogen embrittlement context to quantify the effect of hydrogen on the plastic strain localization at the slip-band scale.

\section{Influence of the subsurface geometry of the grains}

When aiming at modeling explicitly microstructures, it is not always possible to reproduce a real 3D microstructure by volumetric analysis, such as X-ray tomography or destructive metallographic cuts $[7,16,17]$. A common way consists in building a 2-D or extended 2-D mesh from EBSD measurements. In this case, it is important to keep in mind the bias introduced by a simplified mesh generation. When the sample thickness is larger than the grain size, metallurgical studies [18] highlight that the subsurface grain geometry can strongly affect the plasticity in surface. In this part, assuming that the computations using real 3-D meshes are in agreement with the local measured strain fields [7,18], we evaluate the differences between computations using real 3-D and 2-D or extended 2-D meshes when the grain size is close to the thickness of the specimen. The reference microstructures are synthetic 3-D polycrystals of approximately 24 grains modeled and meshed using 3-D voronoi process. Crystal orientation of grains are randomly assigned to each Gauss point. Different set of crystal orientations are tested for each 3-D microstructure. The 2-D meshes are extracted from the grain geometry of each face, and the extended 2D meshes are obtained by extension of the 2-D meshes (Fig. 2) into the thickness direction. Mechanical fields are obtained by finite element calculation using the code ZeBuLoN [19]. Crystal elastoplasticity model based on 12 slip systems for FCC crystal (Table 1) is used to describe the mechanical behavior [20]. The whole equations and the material parameters, determined from experimental tensile test curve, are recalled in the section (3.2).

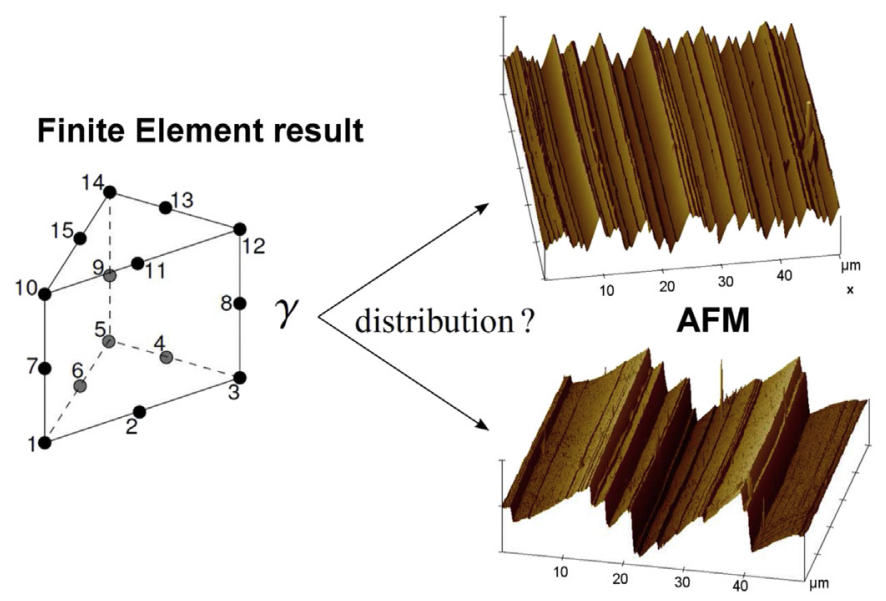

Fig. 1. Numerical result of plastic slip can match several morphologies observed by AFM.

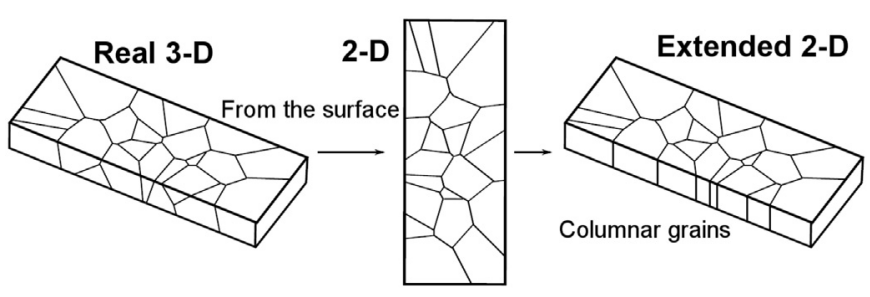

Fig. 2. Methodology of 2-D and extended 2-D mesh generation.

Homogeneous strain boundary conditions are applied to the meshes to simulate a uniaxial tensile test at a constant strain rate of $10^{-3} \mathrm{~s}^{-1}$ up to $2 \%$ plastic strain.

Maps of the cumulated plastic slip $\gamma^{e q}$ at the free surfaces after $2 \%$ plastic strain are showed in Fig. 3. The local cumulative plastic slip is heterogeneous and presents some intense localization zone in which the plastic strain can reach up to six times the imposed deformation. Compared to the real 3-D microstructure, the plastic slip heterogeneities of the extended 2-D mesh are located at the same place. In this case, the mean relative error is about 25\%. But for the 2-D mesh, the plastic localization zones are different and the mean cumulated plastic slip error is about $100 \%$ relative to the $3-\mathrm{D}$ polycrystal.

The maps of the most activated slip plane and of the number of activated planes are drawn in Fig. 4, and 5. The number of activated planes $\left(n_{P}^{a c t}\right)$ is determined according to the Cailletaud's definition of a $\ll$ significantly activated system $\gg[21]$ transposed to the slip plane. If the plastic slip by slip plane exceeds $10 \%$ the maximum slip value of all slip planes, the plane is considered as significantly activated (Fig. 5).

$n_{P}^{a c t}=\sum_{i=1}^{4} H\left(\gamma_{P_{i}}-0.1 \gamma_{P}^{\max }\right)$

with $\gamma_{P_{i}}$ the plastic slip on the plane $P^{i}$ defined by the sum of all plastic slips on the slip plane, and $\gamma_{P}^{\max }$ the maximum slip value of all slip planes.According to the maps of the most activated slip plane and of the number of activated planes, there is a good agreement between the results obtain with the real 3-D mesh and the columnar microstructure. On the contrary, for half of the surface grains, the index of the most activated slip system given by the 2-D modeling is different from one's obtained with the real 3-D microstructure. It is of clear evidence that a 2-D modeling cannot provide accurately, neither the local plastic strain at the surface, neither the activated slip plane. Therefore, when the grain size is close to the sample thickness, using an extruded microstructure is appropriate to represent the surface phenomena, both the most activated plane and the number of slip systems but also the plastic strain level.

Table 1

Slip directions, slip planes and slip systems for FCC metals.

\begin{tabular}{|c|c|c|c|c|c|c|}
\hline Plane & $P^{1}$ & & & $P^{2}$ & & \\
\hline Normal & $\left(\begin{array}{lll}1 & 1 & 1\end{array}\right)$ & & & $(1 \overline{1} 1)$ & & \\
\hline System & $S_{1}^{1}$ & & & $S_{1}^{2}$ & & \\
\hline Direction & {$\left[\begin{array}{lll}\frac{1}{1} & 1 & 1\end{array}\right]$} & {$\left[\begin{array}{lll}0 & \overline{1} & 1\end{array}\right]$} & {$\left[\begin{array}{lll}\frac{3}{1} & 1 & 0\end{array}\right]$} & {$\left[\begin{array}{lll}\frac{1}{1} & 0 & 1\end{array}\right]$} & {$\left[\begin{array}{lll}0 & 1 & 1\end{array}\right]$} & {$\left[\begin{array}{lll}1 & 1 & 0\end{array}\right]$} \\
\hline Plane & & & & $P^{4}$ & & \\
\hline Normal & $\left(\begin{array}{lll}1 & 1 & 1\end{array}\right)$ & & & $\left(\begin{array}{lll}1 & 1 & \overline{1}\end{array}\right)$ & & \\
\hline System & & & & $S_{1}^{4}$ & $S_{2}^{4}$ & $S_{3}^{4}$ \\
\hline Direction & {$\left[\begin{array}{lll}0 & \overline{1} & 1\end{array}\right]$} & {$\left[\begin{array}{lll}1 & 1 & 0\end{array}\right]$} & {$\left[\begin{array}{lll}1 & 0 & 1\end{array}\right]$} & {$\left[\begin{array}{lll}\frac{1}{1} & 1 & 0\end{array}\right]$} & {$\left[\begin{array}{lll}1 & 0 & 1\end{array}\right]$} & {$\left[\begin{array}{lll}0 & 1 & 1\end{array}\right]$} \\
\hline
\end{tabular}



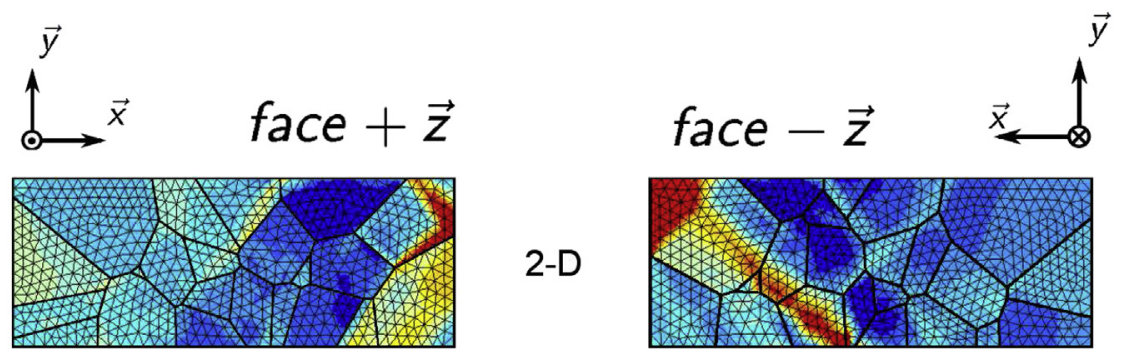

2-D
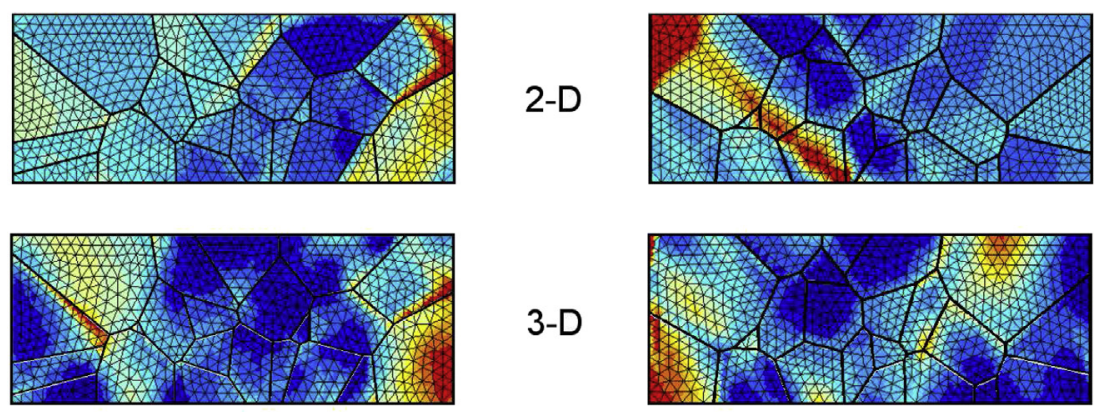

3-D
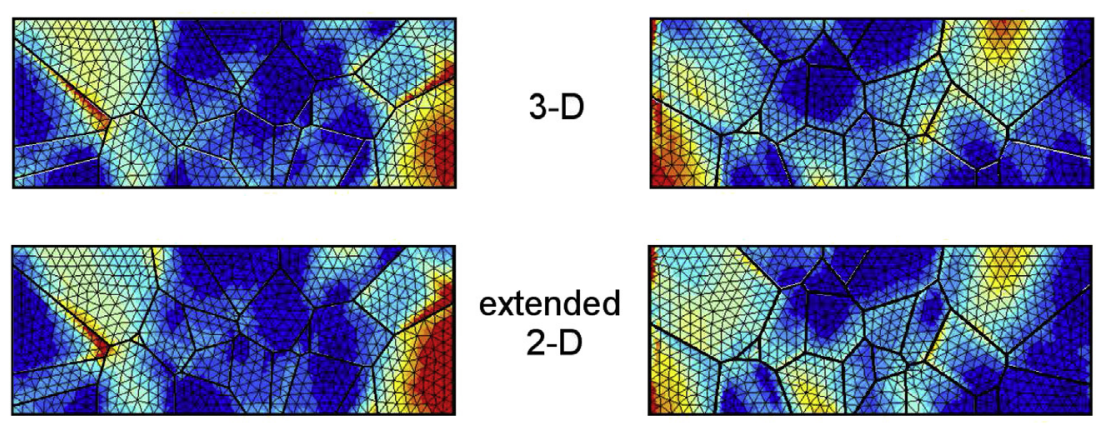

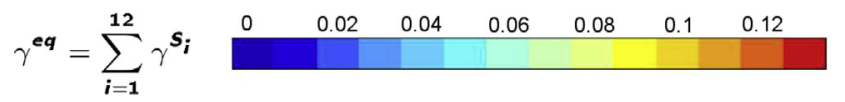

Fig. 3. Cumulated plastic slip at the free surfaces (upper and lower surfaces, respectively) after 2\% plastic strain for the 2-D, 3-D and extended 2-D meshes.
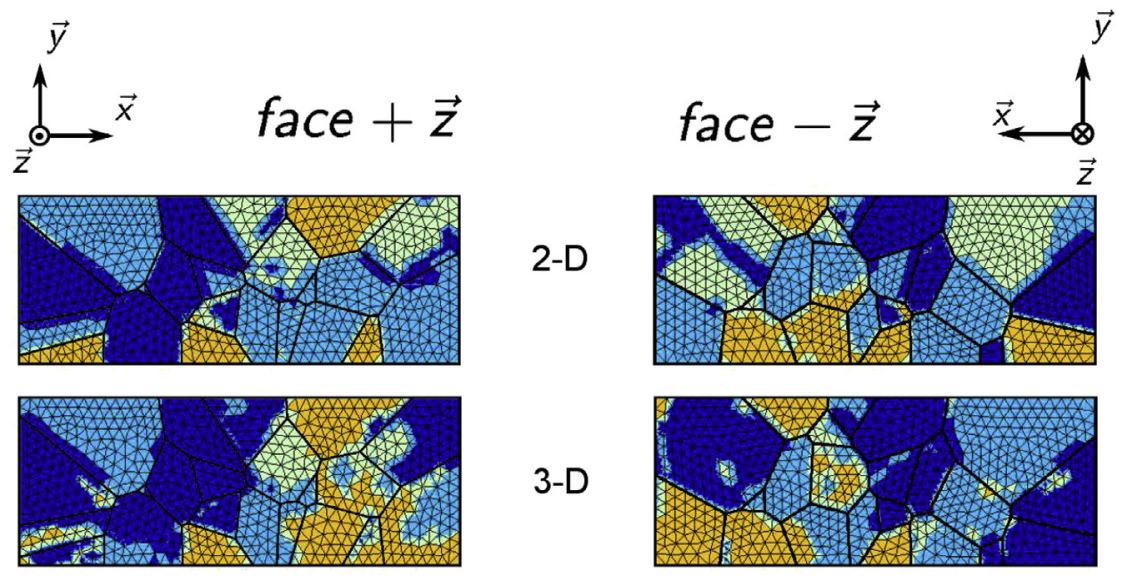

3-D
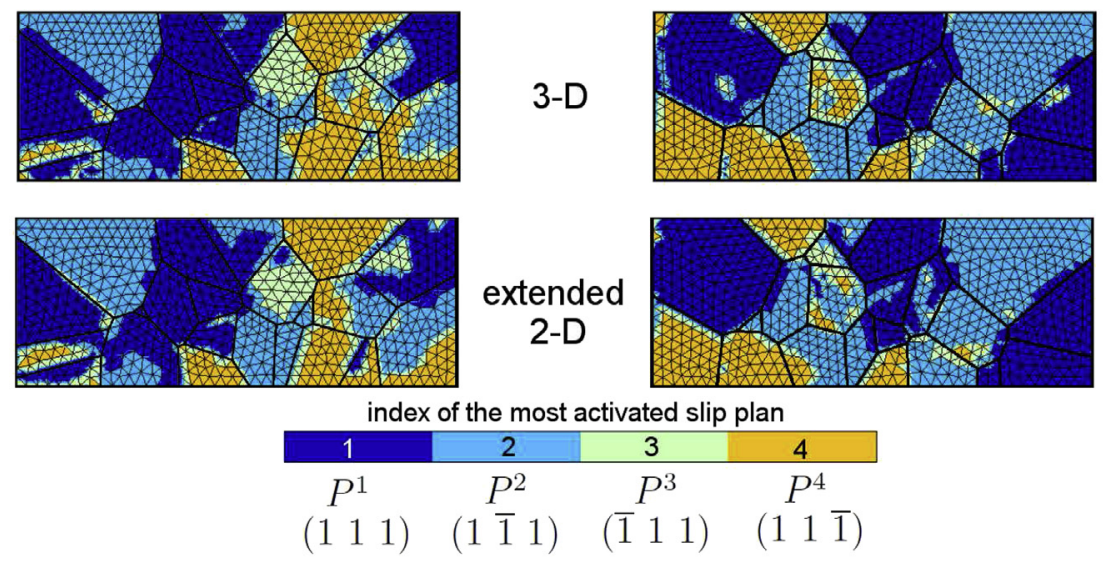

Fig. 4. Index of the most activated slip plane at the free surfaces (upper and lower surfaces, respectively) after 2\% plastic strain for the 2-D, 3-D and extended 2-D meshes.

\section{Methods}

\subsection{Experimental method}

An AISI 316L austenitic stainless steel having an homogeneous coarse microstructure with an equiaxial grain size of $300 \mu \mathrm{m}$ is used. Uniaxial tensile tests are performed in air, at room temperature, and at a constant strain rate of $10^{-3} \mathrm{~s}^{-1}$ up to $3.5 \%$ plastic strain [22]. Electron Back-Scattering Diffraction (EBSD) analysis provides a map of the crystallographic orientations of the investigated area of $2000 \times 3000 \mu \mathrm{m}$ (EBSD analysis step: $14 \mu \mathrm{m})$ and is used to build the numerical model. The deformed sample surfaces 

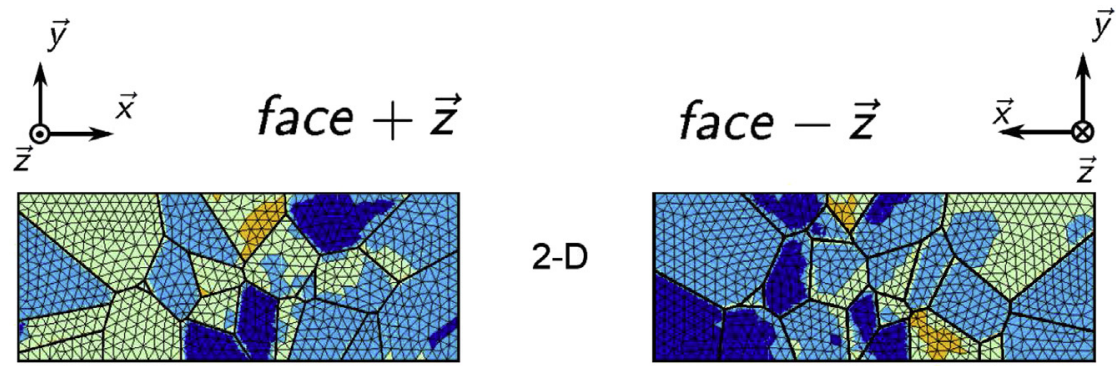

2-D
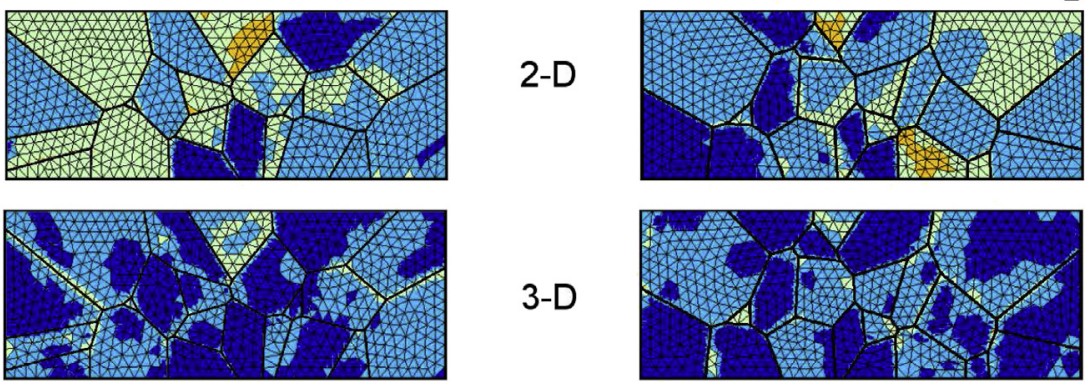

3-D
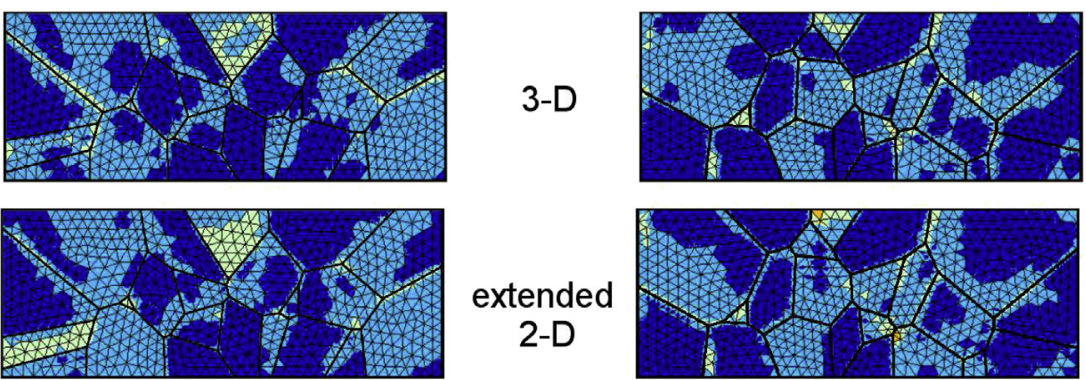

Number of activated slip plan

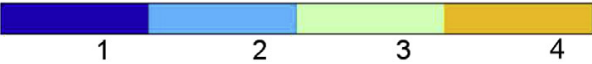

Fig. 5. Number of activated slip plane at the free surfaces (upper and lower surfaces, respectively) after 2\% plastic strain for the 2-D, 3-D and extended 2-D meshes.

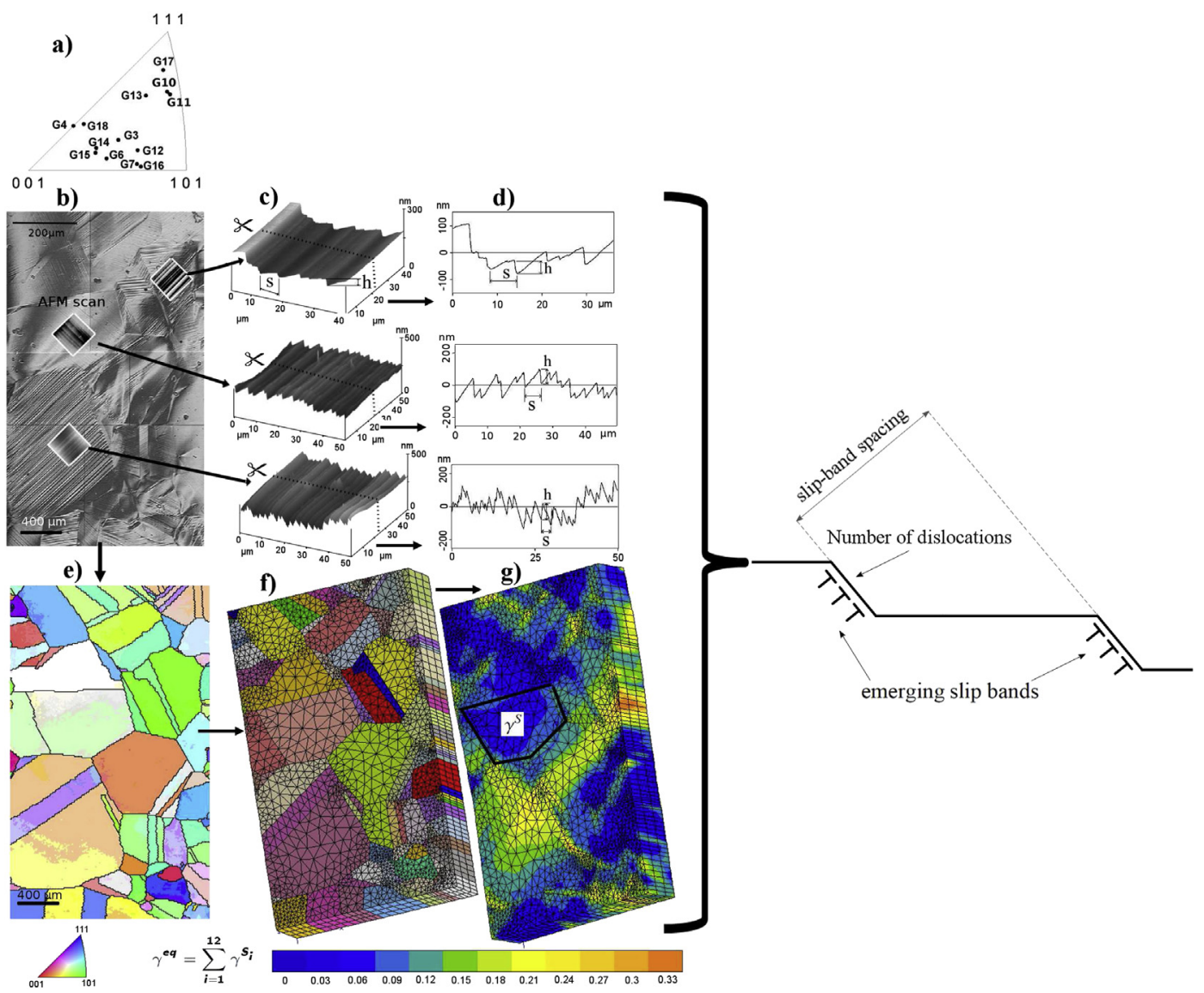

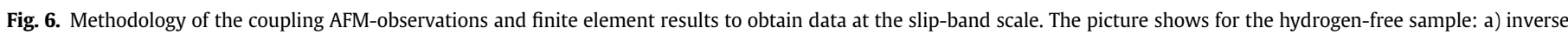
Pole of the observed grains, b) optic image of the strained sample, c) 3D AFM images, d) AFM cross-sections, e) EBSD map, f) mesh and g) numerical cumulated plastic slip. 
are characterized by AFM high resolution topography (VEECO type Dimension 3100) used in contact mode, perpendicularly to the slip bands (Fig. 6a). The scan size is $50 \times 50 \mu \mathrm{m}^{2}$, with 512 pixels per scan line $(0.097 \mu \mathrm{m}$ pixel size). The Atomic Force Microscope being equipped with an optical microscope, AFM scans are performed in the middle of each chosen grain to avoid the grain boundary effect and to observe only primary slip system. Grains showing a too strong heterogeneous deformation are removed from the analyses. For each observed grain, slip-band heights $h$ and slip-band spacings $s$ are measured using an in-house image analysis software from the profiles extracted from the topographical AFM images (Fig. 6). About 12 grains, ie about 500 slip-lines per polycristal are observed to obtain statistically meaningful data. A previous statistical analysis [5] showed that the distributions of $h$ and $s$ were fairly well approximated by a log normal law. The calculation of the meanvalue per grain of $\left\langle s_{A F M}\right\rangle$ and $\left\langle h_{A F M}\right\rangle$ was performed along one AFM-profile by normalizing data with a logarithmic transformation according Neyman and Scott's formula [23]. The Fig. 7 shows that the mean displacement $\overrightarrow{u_{p i}}$ is equal to the sum of the displacements along the three slip directions of this slip plane $\overrightarrow{u_{S_{1}^{i}}}, \overrightarrow{u_{S_{2}}}$ and $\overrightarrow{u_{S i}}$ (Eq. (2)); $S_{j}^{i}$ corresponding to the slip systems related to the slip plane $P^{i}$ and the slip directions $m^{j}$ (j ranging from 1 to 3 ) (see Table 1). The displacement results from the emergence of dislocations having glided on the plane $P^{i}$ and can be expressed as the sum of the mean number of emerged dislocations $\left\langle N_{S_{i}}\right\rangle$ and the Burgers' vector $\overrightarrow{b_{S_{j}^{i}}}$ (Eq. (3)).

$\overrightarrow{u_{P i}}=\overrightarrow{u_{S_{1}^{i}}}+\overrightarrow{u_{S_{2}^{i}}}+\overrightarrow{u_{S_{3}^{i}}}$

$\overrightarrow{u_{S_{j}^{i}}}=\left\langle N_{S_{j}^{i}}\right\rangle \overrightarrow{b_{S_{j}^{i}}}$

For each slip plane $P^{i}$, the mean slip band height $\left\langle h_{P^{i}}\right\rangle$ measured by AFM corresponds to the projection of the displacement $\overrightarrow{u_{P i}}$ on the sample surface normal $\vec{z}$ and is given by:

$$
\begin{aligned}
& \left\langle h_{P^{i}}\right\rangle=\sum_{S_{j}^{i} \in P^{i}}\left|\overrightarrow{u_{S_{j}^{i}}} \cdot \vec{z}\right| \\
& \left\langle h_{P^{i}}\right\rangle=\sum_{S_{j}^{i} \in P^{i}}\left|\left\langle N_{S_{j}^{i}}\right\rangle \overrightarrow{b_{S_{j}^{i}}} \cdot \vec{z}\right|
\end{aligned}
$$

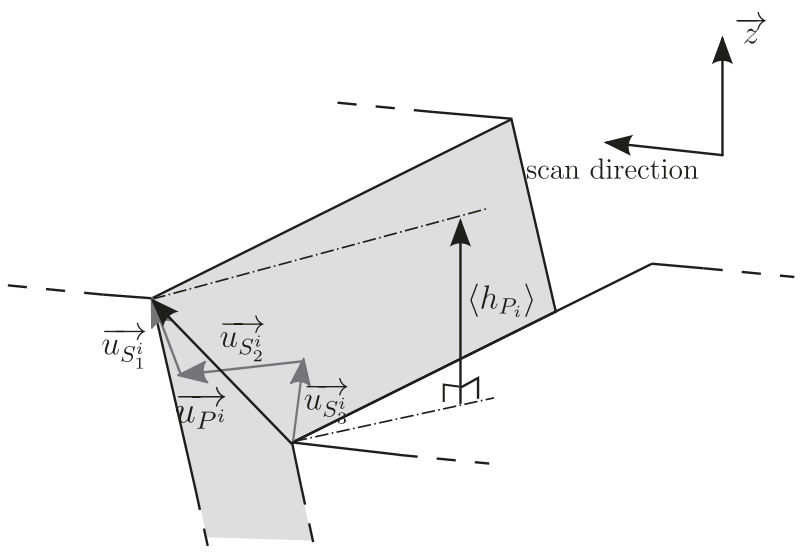

Fig. 7. Link between the mean displacement $\overrightarrow{u_{p i}}$ and the displacements along the three slip directions of this slip plane $\overrightarrow{u_{S_{1}^{i}}}, \overrightarrow{u_{S_{2}}}$ and. $\overrightarrow{u_{S_{3}}}$.

$$
\left\langle h_{P^{i}}\right\rangle=\sum_{S_{j}^{i} \in P^{i}}\left\langle h_{S_{j}^{i}}\right\rangle \text { with }\left\langle h_{S_{j}^{i}}\right\rangle=\left\langle N_{S_{j}^{i}}\right\rangle\left|\overrightarrow{b_{S_{j}^{i}}} \cdot \vec{z}\right|
$$

Thus, a slip band height $\left\langle h_{P^{i}}\right\rangle$ measured by AFM is the sum of the slip band heights $\left\langle h_{S_{i}}\right\rangle$ on each of the three slip systems of the plane $P^{i}$ (Eq. (6)). However, the AFM measurements are not able to determine these values, since no information can be obtained about the slip system activation from those measurements. Therefore, numerical calculations are performed to reach some informations unreachable experimentally.

\subsection{Modeling}

On each grain, plastic slips are determined by finite element computation using the FE code ZeBuLoN [19]. The extended 2-D microstructures are obtained by extruding into the thickness direction, the surface beforehand meshed by free meshing technique with Delaunay triangles from the EBSD data (Fig. 6). Crystal orientations (Euler's angles) and material properties are assigned to each Gauss point. The mechanical behavior of the AISI 316L austenitic stainless steel is described by a crystal elasto-plasticity model as proposed by Cailletaud et al. [20]. Cubic elasticity is used to describe the elastic behavior $\left(C_{1111}=202 \mathrm{GPa}\right.$, $\left.C_{1122}=131 \mathrm{GPa}, C_{1212}=110 \mathrm{GPa}\right)$. At the grain scale, inelastic deformation mainly results of slip on the possible slip systems of the crystal. It is assumed that Schmid's law is valid to compute the resolved shear stress $\tau^{S_{j}^{i}}$ acting on each plane $P^{i}$ in the direction $m^{j}$ (Eq. (7)).

$\tau^{S_{j}^{i}}=\underset{\approx}{\sigma}: \stackrel{S_{j}^{i}}{\underset{\approx}{R}}=\sigma_{k l} R_{k l}^{S_{j}^{i}}$

where $\operatorname{approxR}^{S_{j}^{i}}$ is the orientation tensor of the slip system $S_{j}^{i}$ corresponding to the plane $P^{i}$ and the slip direction $m^{j}$ (Eq. (8)).

$\stackrel{S_{j}^{i}}{\underset{\approx}{\approx}}=\frac{1}{2}\left(\vec{n}^{i} \otimes \vec{m}^{j}+\vec{m}^{j} \otimes \vec{n}^{i}\right)$

With $n^{i}$ the normal of the slip plane $P^{i}$. The shear rate $\dot{\gamma}^{S_{j}^{i}}$ on the slip system with a treshold $\tau_{c}^{S_{j}^{i}}$ is given by (Eq. (9)).

$\dot{\gamma}^{i}=\left(\frac{\left|\tau^{S^{i_{j}}}-x^{S_{j}^{i}}\right|-\tau_{c}^{S_{j}^{i}}}{K}\right)^{n} \operatorname{sign}\left(\tau^{S_{j}^{i}}-x^{S_{j}^{i}}\right)$

where $\mathrm{K}$ and $\mathrm{n}$ characterize the material rate sensitivity, and $x^{S_{j}^{i}}$ the back stress.In this study, only monotonic loading is considered. Consequently, the evolution of the critical resolved shear stress can be described by a nonlinear isotropic hardening law (Eq. (10)).

$\left.\tau_{c}^{S_{j}^{i}}=\tau_{0}^{S_{j}^{i}}+\sum_{r} Q h_{\mathrm{RS}} 1-e^{-b \gamma_{\mathrm{cum}}^{R_{j}^{i}}}\right)$ with $\gamma_{\mathrm{cum}}^{R_{j}^{i}}=\int_{0}^{t}\left|\gamma^{R_{j}^{i}}\right| d t$

The parameters $\tau_{0}^{S_{j}^{i}}$, Q and b are optimized by fitting the results obtained from the finite element calculations and the experimental tensile test curve of the same polycrystal. Crystal plasticity model coefficients are $\mathrm{K}=50 \mathrm{MPa}^{1 / n}, \mathrm{n}=25, \tau_{0}^{S_{j}^{1}}=18.1 \mathrm{MPa}, \mathrm{Q}=85.0$, $\mathrm{b}=1.46$. Self hardening and latent hardening due to interactions between slip systems are introduced by the interaction matrix $h_{R S}$. This matrix was optimized by Guilhem [24] for the AISI 316L stainless steel. $\left(h_{1}=1.0, h_{2}=1.0, h_{3}=0.6, h_{4}=12.3, h_{5}=1.6\right.$, $h_{6}=1.3$ ). Homogeneous strain boundary conditions are applied to 
the extended 2-D mesh to simulate a uniaxial tensile test at a constant strain rate of $10^{-3} \mathrm{~s}^{-1}$ up to a plastic strain of $3.5 \%$

The finite element method (FEM) computations provide the local plastic slip $\gamma^{S_{j}^{i}}$ of each slip system of each grain.

\section{Coupling AFM-observations and finite element calculations}

Experimental mean values $\left\langle h_{A F M}\right\rangle$ and $\left\langle s_{A F M}\right\rangle$ are expressed in the frame of the sample. These scalar values result from the projection of the displacement $\overrightarrow{u_{\text {PAFM }}}$ on the sample surface normal $\vec{z}$ and the scan direction called $\overrightarrow{d_{P A F M}}$, respectively. $\overrightarrow{d_{P A F M}}$ is orthogonal to the slip lines related to the observed slip plane $P^{A F M}$, that will be determined according to Eqs. (16) and (17). On the contrary, numerical results are given in the local frame of the crystal, and have to be expressed in the macroscopic frame according (Eq. (11)).

$\underset{\approx p}{\varepsilon_{j}^{i}}=\gamma_{S_{j}^{i}}: \stackrel{S_{j}^{i}}{\underset{\sim}{R}}$

Where $\underset{\widetilde{\varepsilon}}{S_{j}^{i}}$ is the plastic strain tensor and $\underset{\sim}{S_{j}^{i}}$ is the orientation tensor for $\widetilde{T}^{p}$ e slip system $S_{j}^{i}$ (Eq. (8)).

The plastic strain expressed in the AFM-frame $\gamma_{S_{i}^{i}}^{\text {proj }}$ is calculated by two projections of the strain tensor, firstly on the surface sample normal $\vec{z}$, and then on the scan direction $\overrightarrow{d_{p^{i}}}$ (Eq. (12)).

$\gamma_{S_{j}^{i}}^{p r o j}=\left(\underset{\approx p}{\underset{\approx}{\varepsilon} S_{j}^{i}} \vec{z}\right) \overrightarrow{d_{P^{i}}}=\gamma_{S_{j}^{i}}\left(\underset{\underset{S_{j}^{i}}{R_{z}} \vec{z}}{\approx}\right) \overrightarrow{d_{P^{i}}}$

Considering a region sufficiently close to the sample surface to ensure that all the emerging dislocations from a slip system $S_{j}^{i}$ generate slip bands, the slip $\gamma_{S_{i}^{i}}^{p r o j}$ is related to the mean slip band height $\left\langle h_{S_{j}}\right\rangle$ and spacing $\left\langle S_{S_{j}}\right\rangle$ (see Fig. 8) through the following relation.

$\gamma_{S_{j}^{i}}^{p r o j}=\frac{\left\langle h_{S_{j}^{i}}\right\rangle}{\left\langle s_{S_{j}^{i}}\right\rangle}$

Where $\left\langle h_{S_{i}}\right\rangle$ and $\left\langle s_{S_{i}}\right\rangle$ are the step dimensions measured by AFM if a single system is considered. But, even if there is only one slipband trace, in most cases, the steps are created by more than one slip system according to the same slip plane. The mean values by slip plane of slip-band heights $\left\langle h_{P^{i}}\right\rangle$ and slip-band spacings $\left\langle s_{P^{i}}\right\rangle$ can be connected to the mean values by slip system $\left\langle h_{S_{j}^{i}}\right\rangle,\left\langle s_{S_{j}^{i}}\right\rangle$ according to (Eqs. (6), (13) and (14))

$\left\langle s_{S_{j}^{i}}\right\rangle=\left\langle s_{P^{i}}\right\rangle, \forall S_{j}^{i} \in P^{i}$

The link between the mean value of the slip-band height of a slip plane $P^{i}$, its spacing, and a value $\gamma_{P i}^{p r o j}$ corresponding to the sum of

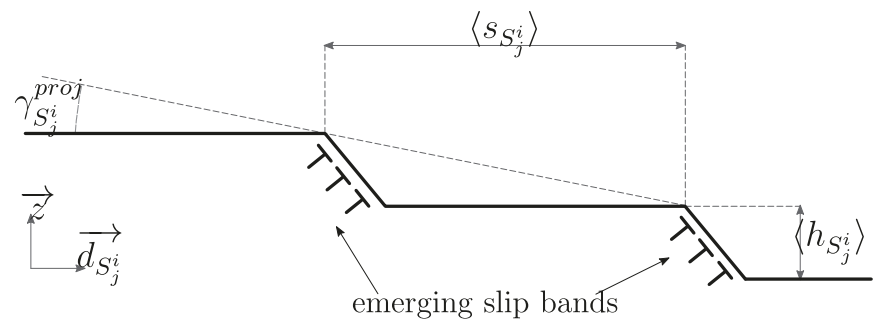

Fig. 8. Link between the mean value of the slip-band height of a slip plane $P^{i}$, its spacing, and a value $\gamma_{p i}^{p r o j}$ corresponding to the sum of the plastic glide on each slip system belonging to the plane $\gamma_{S_{j}^{i}}^{\text {proj }}$. the plastic glide on each slip system belonging to the plane $\gamma_{S_{j}^{i}}^{\text {proj }}$ (Eq. (15)) can be done according (Eq. (14)).

$\left\langle h_{P^{i}}\right\rangle=\sum_{S_{j}^{i} \in P^{i}}\left\langle h_{S_{j}^{i}}\right\rangle=\left\langle s_{P^{i}}\right\rangle \sum_{S_{j}^{i} \in P^{i}} \gamma_{S_{j}^{i}}^{\text {proj }}=\left\langle s_{P^{i}}\right\rangle \gamma_{P^{i}}^{\text {proj }}$

The Eq. (15) is valid whatever the plane $P^{i}$ considered. But the AFM measurements were carried out on the steps corresponding to a specific plane, which is named $P_{A F M}$. To know the slip plane observed by AFM, it is necessary to define the experimental plastic slip $\gamma_{\exp }$ (Eq. (16)).

$\gamma_{\exp }=\frac{\left\langle h_{A F M}\right\rangle}{\left\langle s_{A F M}\right\rangle}$

AFM-observed plane corresponds to the plane which the numerical slip $\gamma_{P_{i}}^{p r o j}$ is closest to the experimental plastic slip $\gamma_{\text {exp }}$, ie which minimizes the relative error $\operatorname{err}_{P^{i}}$ (Eq. (17)). In this case, it was verified that the predicted plane $\gamma_{P i}^{p r o j}$ corresponds to the AFMobserved plane $\gamma_{P i}^{A F M}$ from the angle of visible slip lines on the surface.

$\operatorname{err}_{P^{i}}=\frac{\left|\gamma_{P^{i}}^{\text {proj }}-\gamma_{\exp }\right|}{\gamma_{\exp }}$

If the plane $P^{i}$ matches the plane $P_{A F M}$, the final projection of the AFM results is carried out, involving the experimental results and numerical results.

Thus, the average number of emerging dislocations on the slip system $S_{j}^{i}$ is obtained from (Eq. (6)) and (Eq. (13)) and can be written as:

$\left\langle N_{S_{j}^{i}}\right\rangle=\frac{\left\langle h_{S_{j}^{i}}\right\rangle}{\left|\overrightarrow{b_{S_{j}^{i}}} \cdot \vec{z}\right|}=\frac{\gamma_{S_{j}^{i}}^{p r o j}\left\langle s_{S_{j}^{i}}\right\rangle}{\left|\overrightarrow{b_{S_{j}^{i}}} \cdot \vec{z}\right|}=K_{S_{j}^{i}}^{N}\left\langle s_{S_{j}^{i}}\right\rangle$

with $K_{S_{j}^{i}}^{N}=\frac{\gamma_{S_{j}^{i}}^{p r o j}}{\left|\overrightarrow{b_{S_{j}^{i}}} \cdot \vec{z}\right|}$

The average number of emerging dislocations, $\left\langle N_{P_{A F M}}\right\rangle$ on a plane $P_{A F M}$ is equal to the sum of the numbers of emerging dislocations on each slip system of that plane. According to Eq. (14), we can propose the relation (Eq. (20)) between $\left\langle N_{P_{A F M}}\right\rangle$, the slip-band spacing $\left\langle s_{P_{A F M}}\right\rangle$, and the numerical results through $K_{P_{A F M}}^{N}$ computed from the parameter $\gamma_{P^{i}}^{p r o j}$.

$\left\langle N_{P_{A F M}}\right\rangle=\sum_{S_{j}^{i} \in P_{A F M}}\left\langle N_{S}\right\rangle_{j}^{i}=\sum_{S_{j}^{i} \in P_{A F M}} K_{S_{j}^{i}}^{N}\left\langle s_{S_{j}^{i}}\right\rangle=K_{P_{A F M}}^{N}\left\langle s_{P_{A F M}}\right\rangle$

with $K_{P_{A F M}}^{N}=\sum_{S_{j}^{i} \in P_{A F M}} K_{S_{j}^{i}}^{N}$

According to Fig. 9, the average AFM-observed slip-band spacing $\left\langle s_{P_{A F M}}\right\rangle$ is projected to obtain the real mean value of spacing between the slip planes named $\left\langle s_{P_{A F M}}^{p r o j}\right\rangle$ (Eq. (22)).

$\left\langle s_{P_{A F M}}^{p r o j}\right\rangle=\left\langle s_{P_{A F M}}\right\rangle\left|\overrightarrow{n_{P_{A F M}}} \wedge \vec{z}\right|=\left\langle s_{P_{A F M}}\right\rangle K_{P_{A F M}}^{S}$

Thus, this method is able to determine, at the local discrete scale, not only the real mean slip-band spacing taking into account 


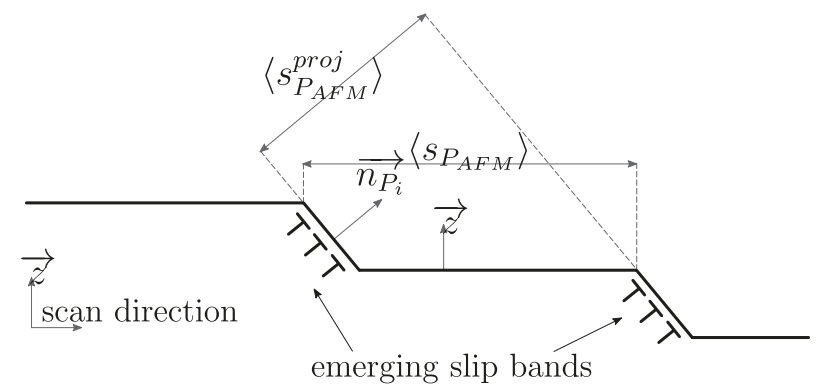

Fig. 9. AFM-observed slip-band spacing $\left\langle s_{P_{A F M}}\right\rangle$ projection to obtain the real mean value $\left\langle s_{P_{A F M}}^{\text {proj }}\right\rangle$ between the slip planes.

the slip plane orientation, but also the number of dislocations having emerged during the plastic strain. The proposed approach needs results from finite element calculations based on EBSD data providing informations about the surface sample microstructure (grain geometry and crystallographic orientations), and from AFMobservations performed after plastic deformation. In the next section, the method is used to quantify the effect of hydrogen on the plastic strain localization.

\section{Application: effect of absorbed hydrogen on emerging dislocations}

Hydrogen-free and hydrogen-charged specimens of AISI 316L stainless steel are deformed by uniaxial tensile tests, in air, at room temperature, and at a constant strain rate of $10^{-3} \mathrm{~s}^{-1}$ up to $3.5 \%$ plastic strain. The average hydrogen concentration $\left(C_{0}\right)$ in the uncharged samples (blank specimens) is $3.5 \pm 0.3$ wt.ppm, whereas the average hydrogen concentration after galvanostatic cathodic charging in $0.1 \mathrm{M} \mathrm{NaOH}$ at $95^{\circ} \mathrm{C}$ and $100 \mathrm{~mA} / \mathrm{cm}^{2}$ for 6 days is

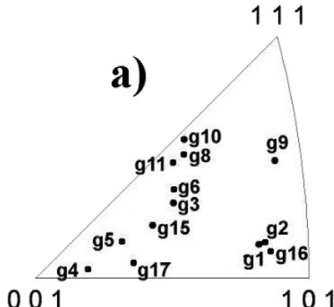

b)
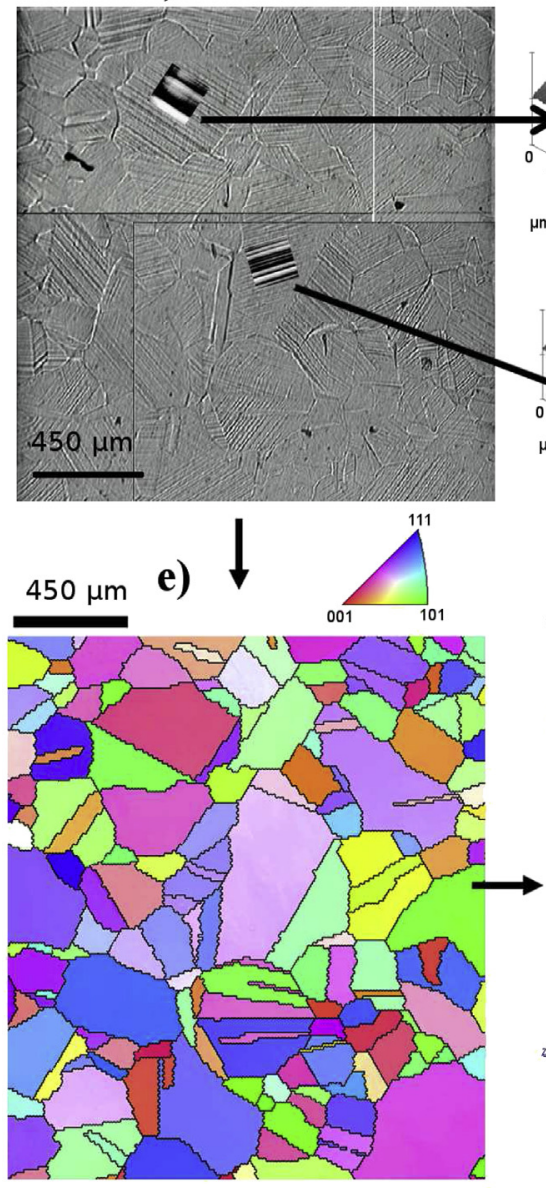

c)

d)
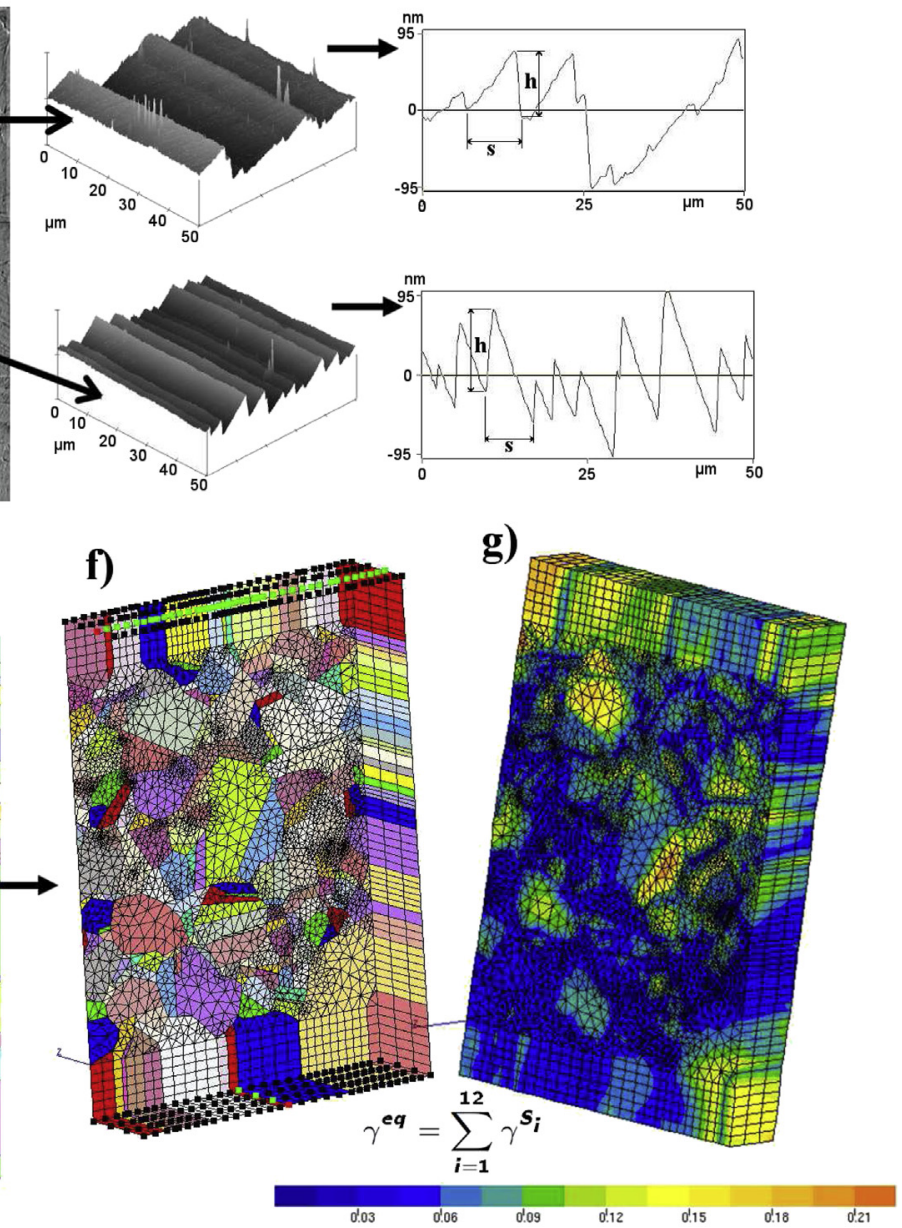

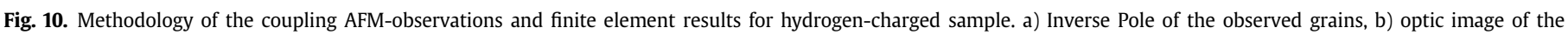
strained sample, c) 3D AFM images, d) AFM cross-sections, e) EBSD map, f) mesh and g) numerical cumulated plastic slip. 
$135 \pm 5$ wt.ppm (see Ref. [25] for details of the experimental methods). A previous statistical analysis based on the observation of approximately 8000 slip bands on 12 specimens [5], showed that, at the polycristal scale, a significant effect of hydrogen on the surface slip morphology was found with these experimental conditions.

Hydrogen-free and hydrogen-charged polycrystals are tested, observed and modeled (Figs. 6 and 10, respectively). The calculation of the experimental plastic slips $\gamma_{\exp }$ are performed from $\left\langle h_{A F M}\right\rangle$ and $\left\langle s_{A F M}\right\rangle$ (Eq. (16)), the mean-values per grain of the slip-band heights $h$ and slip-band spacings $s$ along an AFM-profile at the center of the grain. And the numerical plastic slips are averaged on the elements at the grain center. The minimization of the relative error (Eq. (17)) provides the prediction of the experimentally observed plane (Fig. 11). It was noted a good agreement between the predicted plane and the AFM-observed plane, suggesting that the proposed method of coupling is relevant. Depending on the grains, the relative error ranks between $2 \%$ to $95 \%$, which is rather significant. Several points can explain these differences:

- Although located in the center of the grain, the zones used for averaging the numerical slips are slightly larger than those used for the experimental values. Due to strain heterogeneities, the mean value could be affected.

- Depending on the direction of sliding, the displacement $\overrightarrow{u_{P i}}$ (Fig. 7) may vary along the slip-band. If a slip-band is not visible enough, the measurement of the slip-band spacings can be overvalued. This could explain the greater error for small deformations.

- Using a refined finite element mesh and taking into account the real 3D microstructure could improve the results for smallest grains.

Nevertheless, the scattering of the relative error is the same for hydrogen-free and hydrogen-charged grains, and it is independent of the plastic localization (Fig. 12a) and $12 \mathrm{~b}$ )). We can conclude that the hydrogen effect highlighted in this study is relevant.

At $3.5 \%$ macroscopic strain, Fig. 12a) and b) present, the mean spacing between slip plane $\left\langle s_{P_{A F M}}^{\text {proj }}\right\rangle$ and the mean value of the number of dislocation per slip-band $N_{P_{A F M}}$, respectively, as a function of the plastic slip of the corresponding slip plane $\gamma_{P_{A F M}}$. Each data of Fig. 12 is obtained on one of the grains of each sample. On each graph, light gray and dark gray bars precise the $95 \%$

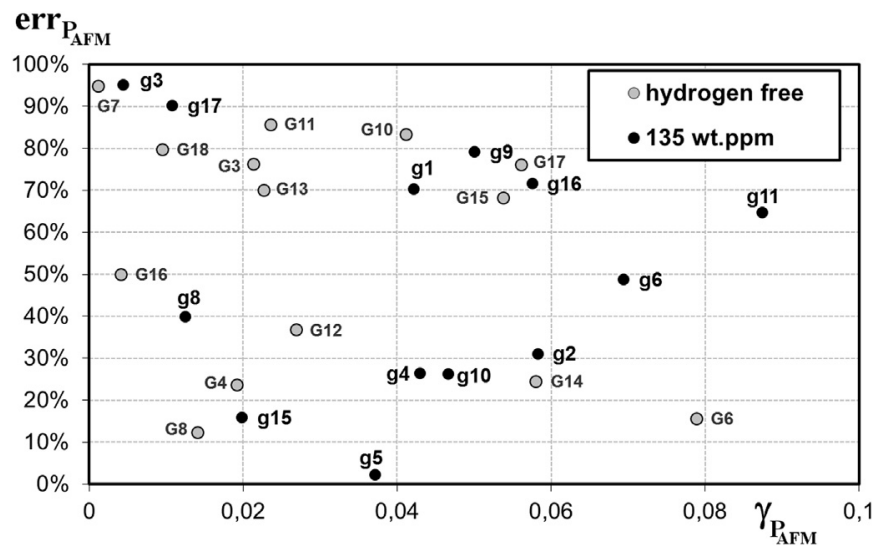

Fig. 11. For each grain, minimal relative error between experimental and numerical plastic slips, as a function of the plastic slip of the corresponding slip plane $\gamma_{P_{A F M}}$.

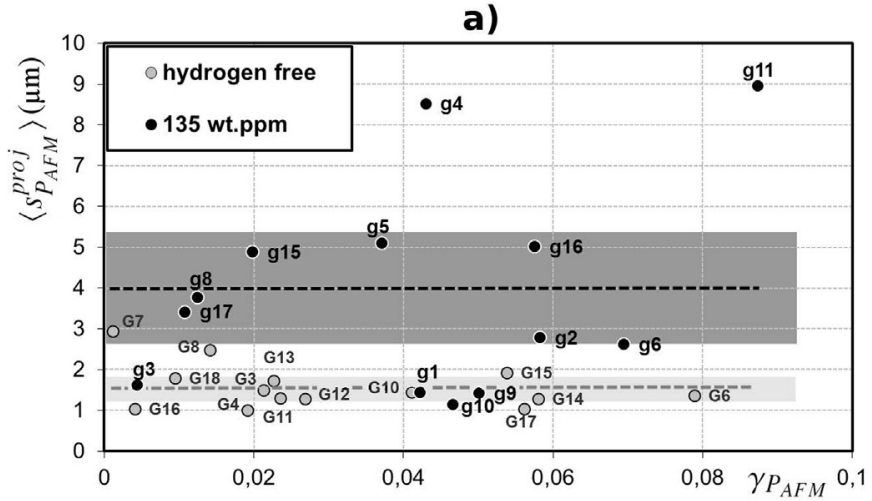

b)

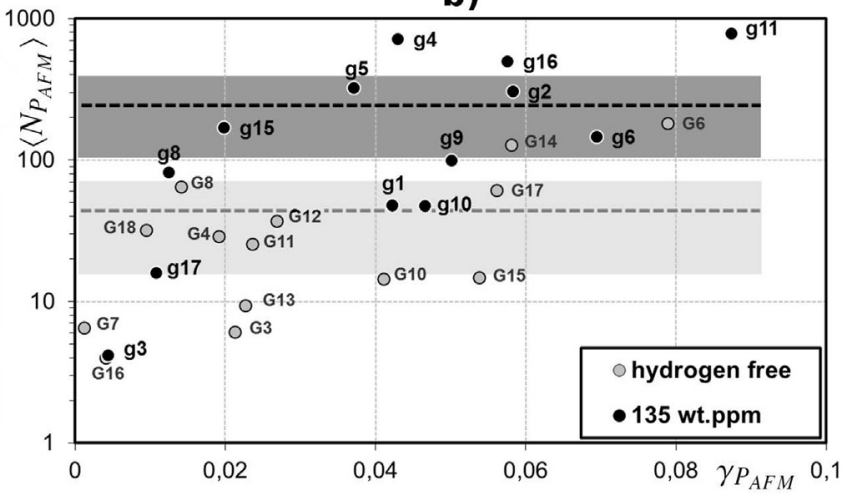

Fig. 12. a) Spacing between slip plane $\left\langle s_{P_{A F M}}^{\text {proj }}\right\rangle$ and b) mean value of the number of dislocation per slip-band $\left\langle N_{P_{A F M}}\right\rangle$, respectively, as a function of the plastic slip of the corresponding slip plane $\gamma_{P_{A F M}}$. Dotted light gray and dark gray lines represent the average values for hydrogen-free and hydrogen-charged samples, respectively. Light gray and dark gray bars precise the $95 \%$ confidence intervals for the hydrogen-free and hydrogen-charged samples, respectively.

confidence intervals for the hydrogen-free and hydrogen-charged samples, respectively. Moreover, after testing the homogeneity of the variances, the calculation of the Student's test (2.85 and 3.32 for $N_{P_{A F M}}$ and $\left\langle s_{P_{A F M}}^{p r o j}\right\rangle$, respectively) shows that the mean values of hydrogen-free and hydrogen-charged data are significantly different at a confidence level of $99.5 \%$. It is clearly shown that $70 \%$ of hydrogenated grains have both a higher $\left\langle N_{P_{A F M}}\right\rangle$ and a larger $\left\langle S_{P_{A F M}}^{p r o j}\right\rangle$ than hydrogen-free grains. Hydrogen effect on plastic localization at the grain scale is clearly evidenced and some hydrogenated grains exhibit a number of dislocation per slip-band up to 50 times higher and a spacing between slip planes 6 times larger than an hydrogen-free grain with the same plastic slip on the observed plane. Nevertheless, for the hydrogen-charged polycrystal, the mean values of the number of dislocation per slip-band and of the spacing between slip planes are 6 times and 2.5 times higher than for the hydrogen free specimen, respectively. So, depending on the grain, a very significant effect of hydrogen absorption on the slip morphology can be highlighted. It is worth noting that these data reveal some scattering due to the complexity of the microstructure (grain shape, crystallographic orientations ...) and to the fact that each grain experiences different stress-strain paths. These results are consistent with the previous ones $[5,25,26]$ that showed an increase of the AFM-observed slip-band spacings and slip-band height with the amount of absorbed hydrogen. In this study, taking into account the crystallographic orientation and the local plastic slip on the observed slip plane highlights a very high plastic strain localization for some grains, which may be critical for the initiation of environment sensitive fracture. 


\section{Conclusion}

In this paper, we propose a new methodology based on finite element crystal plasticity calculations and AFM-observations to obtain data at the slip-band scale. First of all, it is shown that, when the grain size is close to the specimen thickness, using columnar microstructure is suitable to describe the most activated slip plane, the number of slip systems, as well as the plastic strain level on each grain of the polycrystal. This method is able to provide geometrical informations on the slip-bands formed on each grain. The real mean slip-band spacing taking into account the slip plane orientation and the number of dislocations emerging during the plastic strain are obtained. A first application of the method to the effect of hydrogen on the plastic strain localization, allows the quantification of the effect of internal hydrogen on the slip morphology. The data obtained using the presented approach will be useful to link plastic localization and crack initiation in the context of hydrogen embrittlement and stress corrosion cracking.

\section{Acknowledgment}

The authors would like to warmly thank Pr. Xavier Feaugas (Univ. La Rochelle, France) for fruitful discussion and EBSD data, and Pr. Anne-Marie Brass (CNRS) for cooperation.

\section{References}

[1] E. Herms, J.M. Olive, M. Puiggali, Hydrogen embrittlement of 3161 type stainless steel, Mater. Sci. Eng. A 272 (2) (1999) 279-283.

[2] Dislocations in Solids, Hydrogen Effects on Plasticity, 2009. Ch. (chapter 91).

[3] G. Girardin, C. Huvier, D. Delafosse, X. Feaugas, Correlation between dislocation organization and slip bands: TEM and AFM investigations in hydrogencontaining nickel and nickel-chromium, Acta Mater. 91 (2015) 141-151.

[4] C. Huvier, E. Conforto, D. D. El Alami, H. and, X. Feaugas, Some correlations between slip band emergence and dislocation pattern, in: International Conference on Dislocations.

[5] I. Aubert, J.-M. Olive, N. Saintier, The effect of internal hydrogen on surface slip localisation on polycrystalline aisi 3161 stainless steel, Mater. Sci. Eng. A 527 (21-22) (2010) 5858-5866.

[6] R. Miresmaeili, N. Saintier, H. Notsu, J.-M. Olive, H. Kanayama, One-way coupled crystal plasticity-hydrogen diffusion simulation on artificial microstructure, J. Comput. Sci. Technol. 4 (2) (2010) 105-119.

[7] A. Musienko, A. Tatschl, K. Schmidegg, O. Kolednik, R. Pippan, G. Cailletaud, Three-dimensional finite element simulation of a polycrystalline copper specimen, Acta Mater. 55 (12) (2007) 4121-4136.
[8] S. Fréchard, F. Martin, C. Clément, J. Cousty, AFM and EBSD combined studies of plastic deformation in a duplex stainless steel, Mater. Sci. Eng. A 418 (1-2) (2006) 312-319.

[9] C. Coupeau, J. Grilhe, Quantitative analysis of surface effects of plastic deformation, Mater. Sci. Eng. A 271 (1-2) (1999) 242-250.

[10] A. Brinck, C. Engelke, H. Neuhauser, Quantitative AFM investigation of slip line structure in fe3al single crystals after deformation at various temperatures, Mater. Sci. Eng. A 258 (1-2) (1998) 37-41.

[11] M. Risbet, X. Feaugas, Some comments about fatigue crack initiation in relation to cyclic slip irreversibility, Eng. Fract. Mech. 75 (11) (2008) 3511-3519.

[12] D. Chandrasekaran, M. Nygårds, A study of the surface deformation behaviour at grain boundaries in an ultra-low-carbon steel, Acta Mater. 51 (18) (2003) 5375-5384.

[13] A.J. Wilkinson, G. Meaden, D.J. Dingley, High-resolution elastic strain measurement from electron backscatter diffraction patterns: new levels of sensitivity, Ultramicroscopy 106 (4-5) (2006) 307-313.

[14] H. Paul, C. Maurice, J. Driver, Microstructure and microtexture evolution during strain path changes of an initially stable cu single crystal, Acta Mater. 58 (8) (2010) 2799-2813.

[15] Y. Yang, L. Wang, T.R. Bieler, P. Eisenlohr, M.A. Crimp, Quantitative afm characterization and crystal plasticity finite element modeling of heterogeneous deformation in commercial purity titanium, Metall. Mater. Trans. 42 (2011) 636-644.

[16] Y. Bhandari, S. Sarkar, M. Groeber, M. Uchic, D. Dimiduk, S. Ghosh, 3d polycrystalline microstructure reconstruction from FIB generated serial sections for FE analysis, Comput. Mater. Sci. 41 (2) (2007) 222-235.

[17] H. Singh, A.M. Gokhale, Visualization of three-dimensional microstructures, Mater. Charact. 54 (1) (2005) 21-29.

[18] A. Zeghadi, F. N'guyen, S. Forest, A.F. Gourgues, O. Bouaziz, Ensemble averaging stress-strain fields in polycrystalline aggregates with a constrained surface microstructure - part 2: crystal plasticity, Philos. Mag. 87 (8) (2007) 1425-1446.

[19] ZeBuLoN, www.zset-software.com/products/zebulon/.

[20] G. Cailletaud, O. Diard, F. Feyel, S. Forest, Computational crystal plasticity: from single crystal to homogenized polycrystals, Tech. Mech. 23 (2003) $130-145$.

[21] G. Cailletaud, Une approche micromecanique phenomenologique du comportement inelastique des metaux (Ph.D. thesis), Universit Pierre et Marie Curie, Paris 6, 1987.

[22] A.-M. Brass, J. Chêne, Hydrogen uptake in 3161 stainless steel: consequences on the tensile properties, Corros. Sci. 48 (2006) 3222-3242.

[23] J. Neyman, E. Scott, Correction for bias introduced by a transformation of variables, Ann. Math. Stat. 31 (1960) 643-655.

[24] Y. Guilhem, S. Basseville, F. Curtit, J--M. Stéphan, G. Cailletaud, Numerical investigations of the free surface effect in three-dimensional polycrystalline aggregates, Comput. Mater. Sci. 70 (0) (2013) 150-162.

[25] I. Aubert, N. Saintier, J.-M. Olive, Crystal plasticity computation and atomic force microscopy analysis of the internal hydrogen-induced slip localization on polycrystalline stainless steel, Scr. Mater. 66 (9) (2012) 698-701.

[26] D. Delafosse, X. Feaugas, I. Aubert, N. Saintier, J. Olive, Hydrogen effects on the plasticity of fcc nickel and austenitic alloys, in: Proceedings of the 2008 International Hydrogen Conference - Effects of Hydrogen on Materials. 